Internet Engineering Task Force (IETF)

A. Morton

Request for Comments: 6038

Updates: 5357

Category: Standards Track

Ciavattone

AT\&T Labs

ISSN : 2070-1721

Two-Way Active Measurement Protocol (TWAMP) Reflect Octets and Symmetrical Size Features

Abstract

This memo describes two closely related features for the core specification of the Two-Way Active Measurement Protocol (TWAMP): an optional capability where the responding host returns some of the command octets or padding octets to the sender, and an optional sender packet format that ensures equal test packet sizes are used in both directions.

Status of This Memo

This is an Internet Standards Track document.

This document is a product of the Internet Engineering Task Force (IETF). It represents the consensus of the IETF community. It has received public review and has been approved for publication by the Internet Engineering Steering Group (IESG). Further information on Internet Standards is available in section 2 of RFC 5741.

Information about the current status of this document, any errata, and how to provide feedback on it may be obtained at http://www.rfc-editor.org/info/rfc6038.

Copyright Notice

Copyright (c) 2010 IETF Trust and the persons identified as the document authors. All rights reserved.

This document is subject to BCP 78 and the IETF Trust's Legal Provisions Relating to IETF Documents

(http://trustee.ietf.org/license-info) in effect on the date of publication of this document. Please review these documents carefully, as they describe your rights and restrictions with respect to this document. Code Components extracted from this document must include Simplified BSD License text as described in Section $4 . e$ of the Trust Legal Provisions and are provided without warranty as described in the Simplified BSD License. 
This document may contain material from IETF Documents or IETF Contributions published or made publicly available before November 10, 2008. The person(s) controlling the copyright in some of this material may not have granted the IETF Trust the right to allow modifications of such material outside the IETF Standards Process. Without obtaining an adequate license from the person(s) controlling the copyright in such materials, this document may not be modified outside the IETF Standards Process, and derivative works of it may not be created outside the IETF Standards Process, except to format it for publication as an RFC or to translate it into languages other than English.

Table of Contents

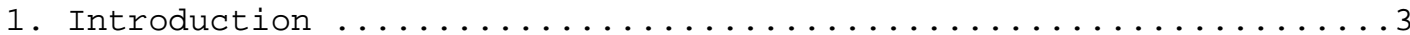

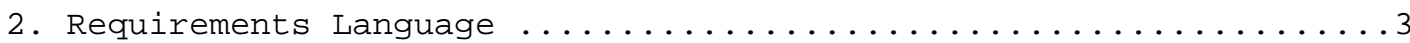

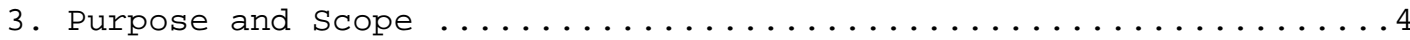

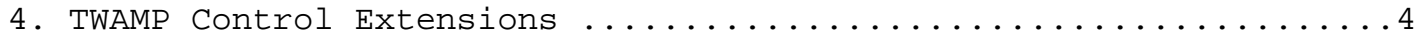

4.1. Connection Setup with New Features ..................

4.2. Reflect Octets: Request-TW-Session Packet Format ........6

4.3. Reflect Octets: Accept Session Packet Format ............7

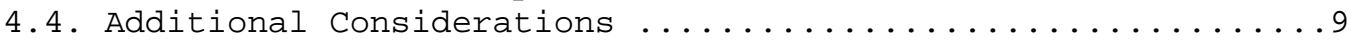

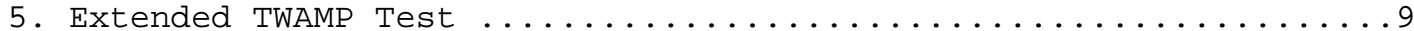

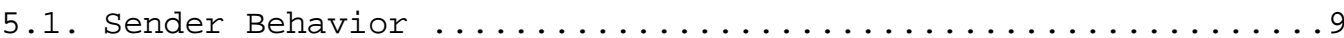

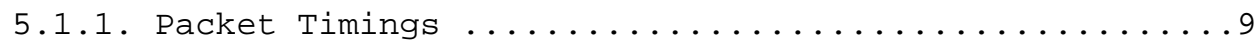

5.1.2. Reflect Octets: Packet Formats and Contents .......9

5.1.3. Reflect Octets: Interaction with Padding

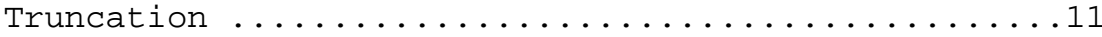

5.1.4. Symmetrical Size: Session-Sender Packet Format ....13

5.1.5. Symmetrical Size AND Reflect Octets:

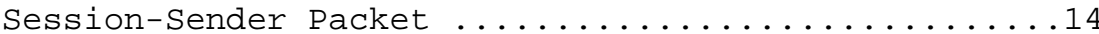

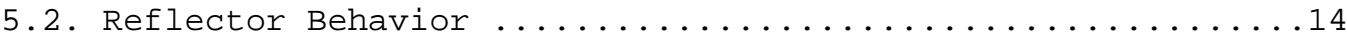

5.2.1. Reflect Octets: Session-Reflector Packet

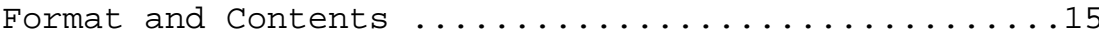

5.2.2. Symmetrical Size: Session-Reflector Packet Format ..16 5.2.3. Symmetrical Size AND Reflect Octets:

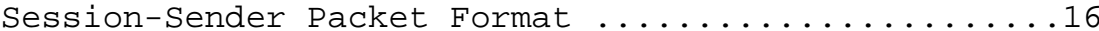

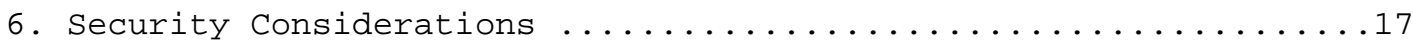

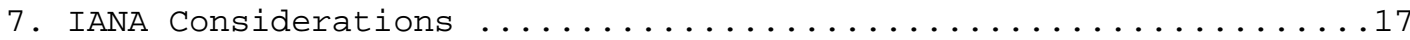

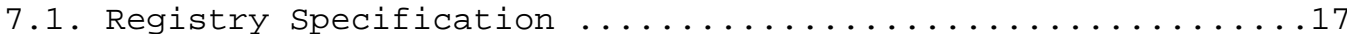

7.2. Registry Contents .......................17

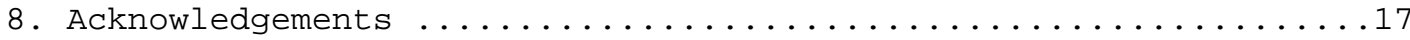

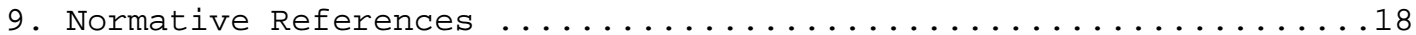


1. Introduction

The Two-Way Active Measurement Protocol (TWAMP) [RFC5357] is an extension of the One-way Active Measurement Protocol (OWAMP) [RFC4656]. The TWAMP specification gathered wide review as it approached completion, and the by-products were several recommendations for new features in TWAMP.

This memo describes two closely related features for TWAMP.

One is the OPTIONAL capability for the responder host to return a limited number of unassigned (padding) octets to the Control-Client or Session-Sender entities in both the TWAMP-Control and TWAMP-Test protocols. With this capability, the Control-Client or sessionSender can embed octets of information it deems useful and have the assurance that the corresponding reply/test packet will contain that information when it is reflected and returned (by the server or Session-Reflector).

The memo also adds an OPTIONAL capability to ensure that reflected test packets are the same size in both directions of transmission. This is accomplished by specifying a new TWAMP-Test session-Sender packet format. Although TWAMP [RFC5357] recommends padding truncation to achieve symmetrical sizes (to compensate for the Session-Reflector's larger test packet header), it's not guaranteed that the Session-Reflector will truncate the padding, and it's not possible if there's insufficient padding.

This memo is an update to the TWAMP core protocol specified in [RFC5357]. Measurement systems are not required to implement the features described in this memo to claim compliance with [RFC5357].

Throughout this memo, the bits marked MBZ (Must Be Zero) MUST be set to zero by senders and MUST be ignored by receivers. Also, the HMAC (Hashed Message Authentication Code) MUST be calculated as defined in Section 3.2 of [RFC4656].

2. Requirements Language

The key words "MUST", "MUST NOT", "REQUIRED", "SHALL", "SHALL NOT", "SHOULD", "SHOULD NOT", "RECOMMENDED", "MAY", and "OPTIONAL" in this document are to be interpreted as described in RFC 2119 [RFC2119]. 


\section{Purpose and Scope}

The purpose of this memo is to define two OPTIONAL closely related features for TWAMP [RFC5357]. The features enhance the TWAMP host's capabilities to perform a simple operation on control and test packets: the reflection of octets or padding, and the capability to ensure symmetrical size TWAMP-Test packets. Motivations include permitting the controller host to tag packets with an index for simplified identification, and/or assert that the same size test packets will be used in each direction.

The scope of the memo is limited to specifications of the following features:

- Reflect Octets: the capability of the Server/Session-Reflector to reflect specific octets back to the client/Session-Sender, and a similar service provided by the Client and Session-Sender.

- Symmetrical Size: the capability to ensure that TWAMP-Test protocol uses the same packet size in both directions through support of a new TWAMP-Test Session-Sender test packet format in both the Session-Sender and the Session-Reflector. Only the Session-Sender test packet format is new.

This memo extends the modes of operation through assignment of two new values in the Modes field (see Section 3.1 of [RFC4656] for the format of the Server Greeting message), while retaining backward compatibility with the core TWAMP [RFC5357] implementations. The two new values correspond to the two features defined in this memo.

When the Server and Control-Client have agreed to use the Reflect octets mode during control connection setup, then the Control-client, the Server, the Session-Sender, and the Session-Reflector MUST all conform to the requirements of that mode, as identified below.

When the Server and Control-client have agreed to use the Symmetrical Size mode during control connection setup, then the Control-Client, the Server, the Session-Sender, and the Session-Reflector MUST all conform to the requirements of that mode, as identified below.

4. TWAMP Control Extensions

TWAMP-Control protocol [RFC5357] uses the Modes field to identify and select specific communication capabilities, and this field is a recognized extension mechanism. The following sections describe two such extensions. 


\subsection{Connection Setup with New Features}

TWAMP connection establishment follows the procedure defined in Section 3.1 of [RFC4656] and Section 3.1 of [RFC5357]. The new features require two new bit positions (and values) to identify the ability of the Server/Session-Reflector to reflect specific octets back to the Control-Client/Session-Sender, and to support the new Session-Sender packet format in the TWAMP-Test protocol. See the IANA section for details on the assigned values and bit positions.

The Server sets one or both of the new bit positions in the Modes field of the Server Greeting message to indicate its capabilities and willingness to operate in either of these modes (or both) if desired.

If the Control-Client intends to operate all test sessions invoked with this control connection using one or both of the new modes, it MUST set the Mode field bit corresponding to each function in the Setup Response message. With this and other extensions, the ControlClient MAY set multiple Mode field bits in the setup Response message. 


\subsection{Reflect Octets: Request-TW-Session Packet Format}

The bits designated for the Reflect Octets feature in the Request-TWSession command are as shown in the packet format below.

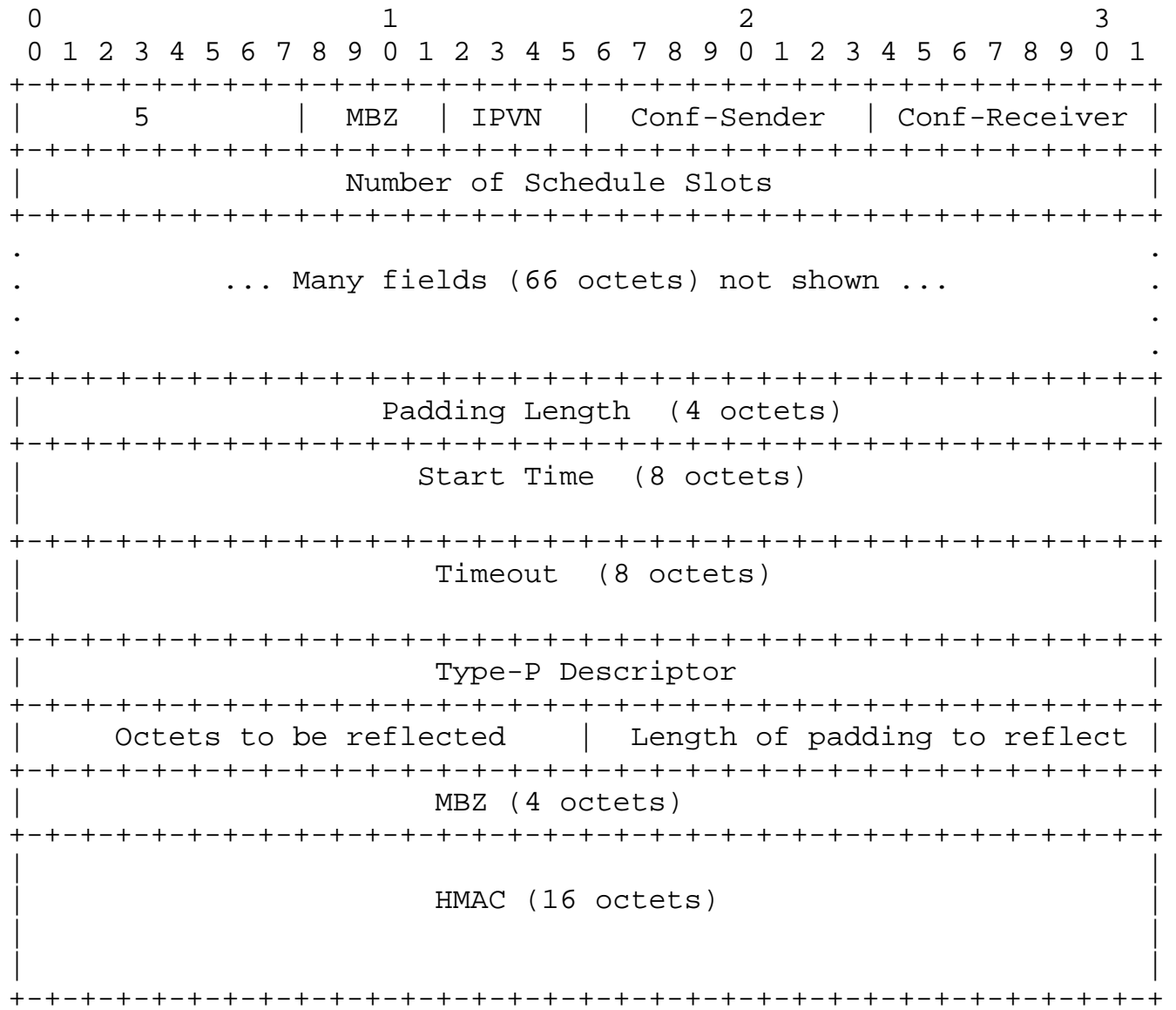

It is important to note that the Padding Length field continues to specify the number of padding octets that the Session-Sender will append to ALL TWAMP-Test packets associated with this test session. See below for considerations on the minimum length of the padding octets following the definitions of the two new fields that follow the Type-P Descriptor.

Note that the number of padding octets appended to the SessionReflector's test packet depends on support for the truncation process that TWAMP recommends, see Section 4.2.1 of [RFC5357]. 
The Octets to be reflected field SHALL be 2 octets long, as shown, and contain the octets that the Server MUST reflect in the Accept Session message as specified below.

The Length of padding to reflect field SHALL be 2 octets long and contain an unsigned binary value in units of octets. This field communicates the length of the padding in the TWAMP-Test Packet that the Session-Sender expects to be reflected and the length of octets that the Session-Reflector SHALL return in its TWAMP-Test packet format (see Section 5.2). By including this length field in the Request-TW-Session message, a Server is able to determine if it can comply with a specific request to reflect padding in the TWAMP-Test packets and to arrange for the Session-Reflector processing in advance.

The Padding Length SHOULD be $>=27$ octets when specifying a test session using the Unauthenticated TWAMP-Test mode to allow for the truncation process that TWAMP recommends, see section 4.2 .1 of [RFC5357].

The Padding Length SHOULD be $>=56$ octets when specifying a test session using the Authenticated or Encrypted TWAMP-Test modes to allow for the truncation process that TWAMP recommends, see section 4.2 .1 of [RFC5357].

The Padding Length SHALL be $>$ the Length of padding to reflect when specifying a test session using the OPTIONAL Reflect Octets mode.

In Unauthenticated TWAMP-Test mode, the Padding Length SHALL be $>=27$ + Length of padding to reflect octets when specifying a test session using both the OPTIONAL Reflect Octets mode and the truncation process that TWAMP recommends, see section 4.2.1 of [RFC5357].

In Authenticated or Encrypted TWAMP-Test modes, the Padding Length SHALL be $>=56$ + Length of padding to reflect octets when specifying a test session using both the OPTIONAL Reflect Octets mode and the truncation process that TWAMP recommends, see section 4.2 .1 of [RFC5357].

\subsection{Reflect Octets: Accept Session Packet Format}

The bits designated for the Reflect Padding feature in the Accept Session command are as shown in the packet format below. 


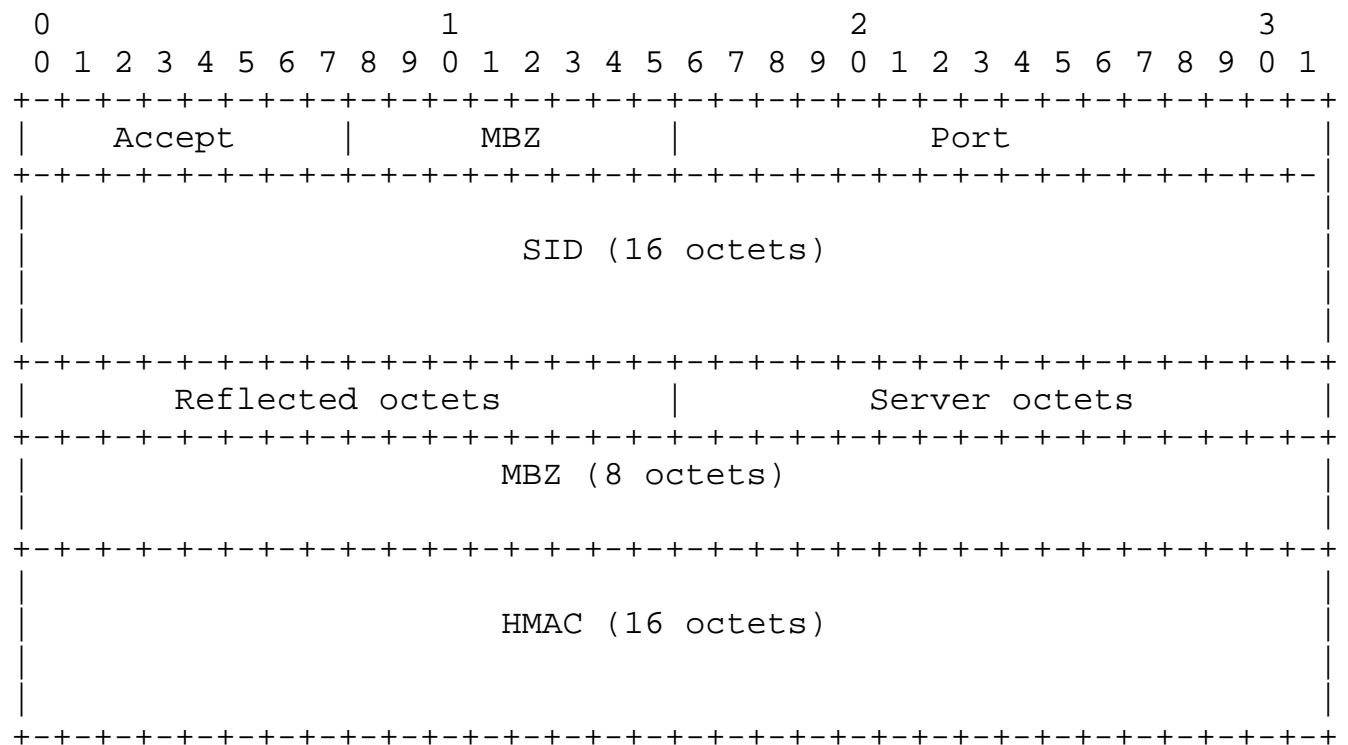

The Reflected octets field SHALL contain the octets from the RequestTW-Session Octets to be reflected field and be 2 octets long, as shown.

The Server octets field SHALL contain information that the Server intends to be returned in the TWAMP-Test Packet Padding (to be reflected) field, OR SHALL be zero, and be 2 octets long, as shown. Although the Server determines the SID, this field is very long (16 octets) and does not normally appear in TWAMP-Test packets. The following items MUST be part of compliant implementations using the Reflect Octets feature:

- When a Server does not require octets to be returned in TWAMP-Test packets, it MUST send all zeros in the server octets field.

- When a Server intends octets to be returned in TWAMP-Test packets, it MUST send a non-zero value in the Server octets field, and the TWAMP-Test Sender MUST include those octets at the beginning of the Packet Padding (to be reflected) field.

- Section 4.1.2 defines how Server octets MUST be included in the TWAMP-Test packet padding when this service is desired by the Server (indicated in the second of two figures in this section). 
When performing the truncation process that TWAMP recommends, see Section 4.2.1 of [RFC5357], if calculations on the Padding lengths reveal that there are insufficient octets supplied to produce equallength Session-Sender and Session-Reflector test packets, then the Accept field MUST be set to 3 to indicate that some aspect of the request is not supported.

\subsection{Additional Considerations}

The value of the Modes field sent by the Server in the Server Greeting message is the bit-wise OR of the mode values that it is willing to support during this session.

With the publication of this memo as an RFC, the last 7 bit positions of the Modes 32-bit field are used. A Control-Client conforming to this extension of [RFC5357] MAY ignore the values in the higher bits of the Modes field, or it MAY support other features that are communicated in those bit positions. The other bits are available for future protocol extensions.

5. Extended TWAMP Test

The TWAMP-Test protocol is similar to the OWAMP [RFC4656] test protocol with the exception that the session-Reflector transmits test packets to the Session-Sender in response to each test packet it receives. TWAMP, see Section 4 of [RFC5357], defines two additional test packet formats for packets transmitted by the Session-Reflector. The appropriate format depends on the security mode chosen. The new modes specified here utilize some of the padding octets within each test packet format or require truncation of those octets depending on the security mode in use.

\subsection{Sender Behavior}

This section describes extensions to the behavior of the TWAMP Session-Sender.

5.1.1. Packet Timings

The Send Schedule is not utilized in TWAMP, and this is unchanged in this memo.

\subsubsection{Reflect Octets: Packet Formats and Contents}

The Session-Sender packet format and content follow the same procedure and guidelines as defined in section 4.1.2 of [RFC4656] (as indicated in section 4.1 .2 of [RFC5357]). 
The Reflect Octets mode re-designates the original TWAMP-Test Packet Padding field (see Section 4.1.2 of [RFC4656]), as shown below for Unauthenticated mode:

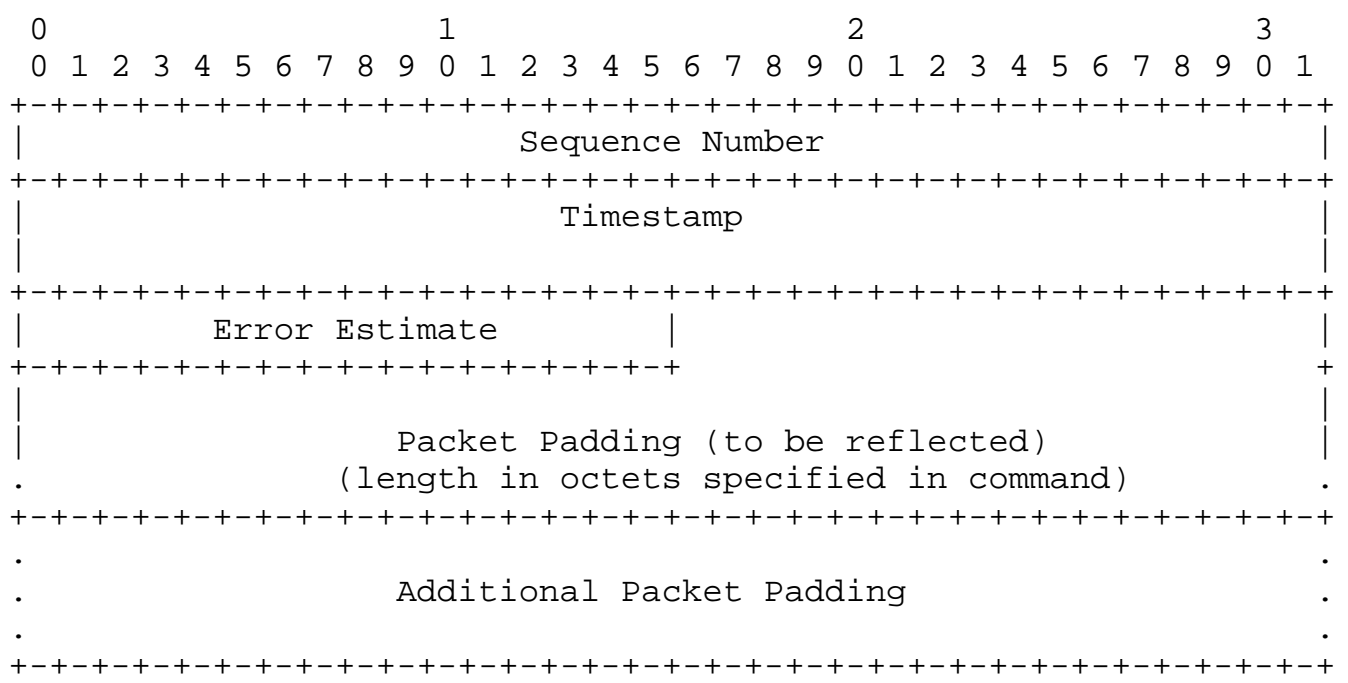

The Packet Padding (to be reflected) field SHALL correspond to the length of octets specified in the Request-TW-Session Length of padding to reflect field to this test session. These are the octets that the Session-Sender expects will be returned by the SessionReflector.

The length of the Additional Packet Padding field is the difference between two fields in the Request-TW-Session command, as follows:

Additional Packet Padding, in octets $=$

Padding Length - Length of padding to reflect

When a Server intends octets to be returned in TWAMP-Test packets, it MUST send a non-zero value in the Server octets field, and the TWAMPTest Session-Sender MUST include those octets in the first 2 octets of the Packet Padding (to be reflected) field as shown below: 


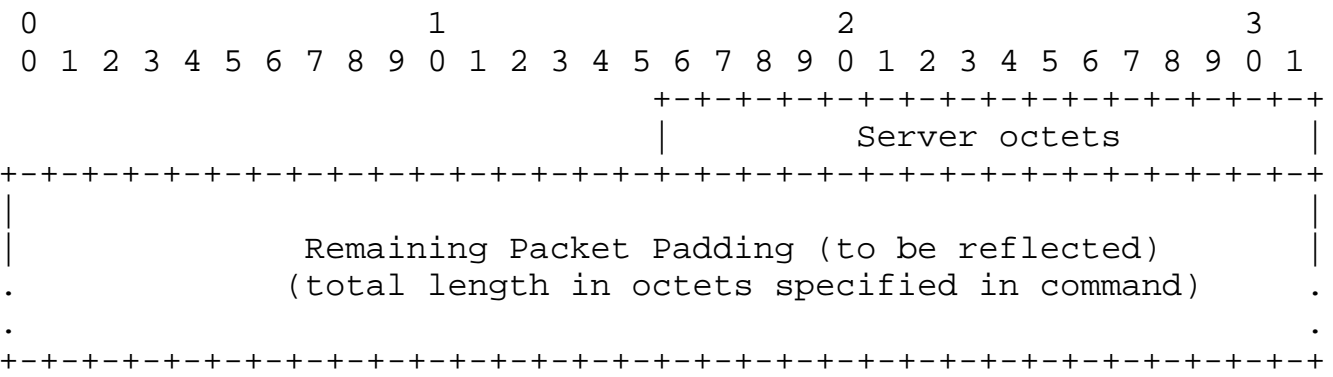

The Server octets field contains the same information that the Server returned to the Control-Client in the Accept-Session message corresponding to this specific test session (see Section 4.3). At the Session-Reflector, these octets MUST be reflected the same as the rest of the Packet Padding (to be reflected) field.

Note that it is permissible for the Session-Sender to insert the same octets used in the Octets to be reflected field of the Request-TWSession command elsewhere in the Packet Padding (to be reflected) field.

\subsubsection{Reflect Octets: Interaction with Padding Truncation}

When the Reflect Octets mode is selected, and performing the truncation process that TWAMP recommends, see section 4.2 .1 of [RFC5357], the Session-Sender MUST anticipate a minimum padding required to achieve equal-size test packets in both directions. The amount of padding needed to achieve symmetrical packet size depends on both the security mode (Unauthenticated/Authenticated/Encrypted) and whether the Reflect Octets mode is selected simultaneously.

When using the truncation process in TWAMP alone, see section 4.2.1 of [RFC5357], the Session-Sender MUST append sufficient Packet Padding octets to allow the same IP packet payload lengths to be used in each direction of transmission (this is usually desirable). To compensate for the Session-Reflector's larger test packet format, the Session-Sender MUST append at least 27 octets of padding in Unauthenticated mode, and at least 56 octets in Authenticated and Encrypted modes. The sizes of TWAMP-Test protocol packets and the resulting truncated padding to achieve equal packet sizes in both directions are shown in the table below: 


\begin{tabular}{|c|c|c|}
\hline Octets in: & Unauthenticated Mode & Auth/Encrypted Mode \\
\hline Roflogtor Hoador & & 101 \\
\hline Retlector Header & 41 & $\begin{array}{l}104 \\
48\end{array}$ \\
\hline Truncated Padding & $\begin{array}{l}14 \\
27\end{array}$ & \\
\hline
\end{tabular}

TWAMP-Test Padding Truncation

When using the Reflect Octets mode simultaneously with the truncation process that TWAMP recommends in section 4.2.1 of [RFC5357], the Session-Sender MUST append at least 27 octets of padding plus the Length of the padding to reflect octets when operating in Unauthenticated mode. The Session-Sender MUST append at least 56 octets of padding plus the Length of the padding to reflect octets when operating in Authenticated and Encrypted modes. 


\subsubsection{Symmetrical Size: Session-Sender Packet Format}

When the Symmetrical Size mode is selected, the Session-Sender SHALL use the following TWAMP-Test Packet Format in Unauthenticated mode:

Unauthenticated Mode

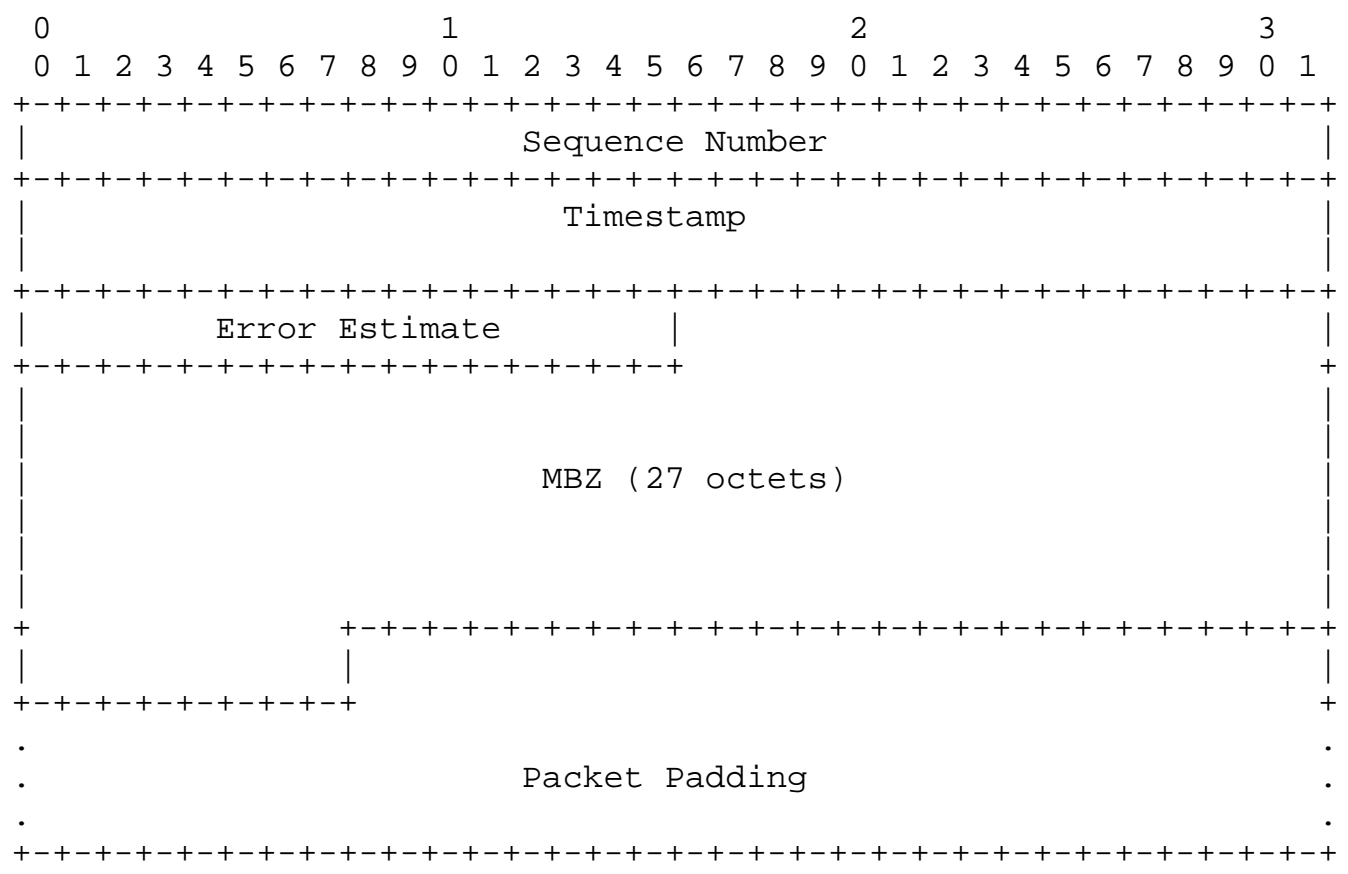

This feature REQUIRES only a new Session-Sender test packet format, the Session-Reflector test packet format is unchanged. 


\subsubsection{Symmetrical Size AND Reflect Octets: Session-Sender Packet Format}

When both the Symmetrical Size mode and the Reflect Octets mode are selected, the Session-Sender SHALL use the following TWAMP-Test Packet Format in Unauthenticated mode:

Unauthenticated Mode

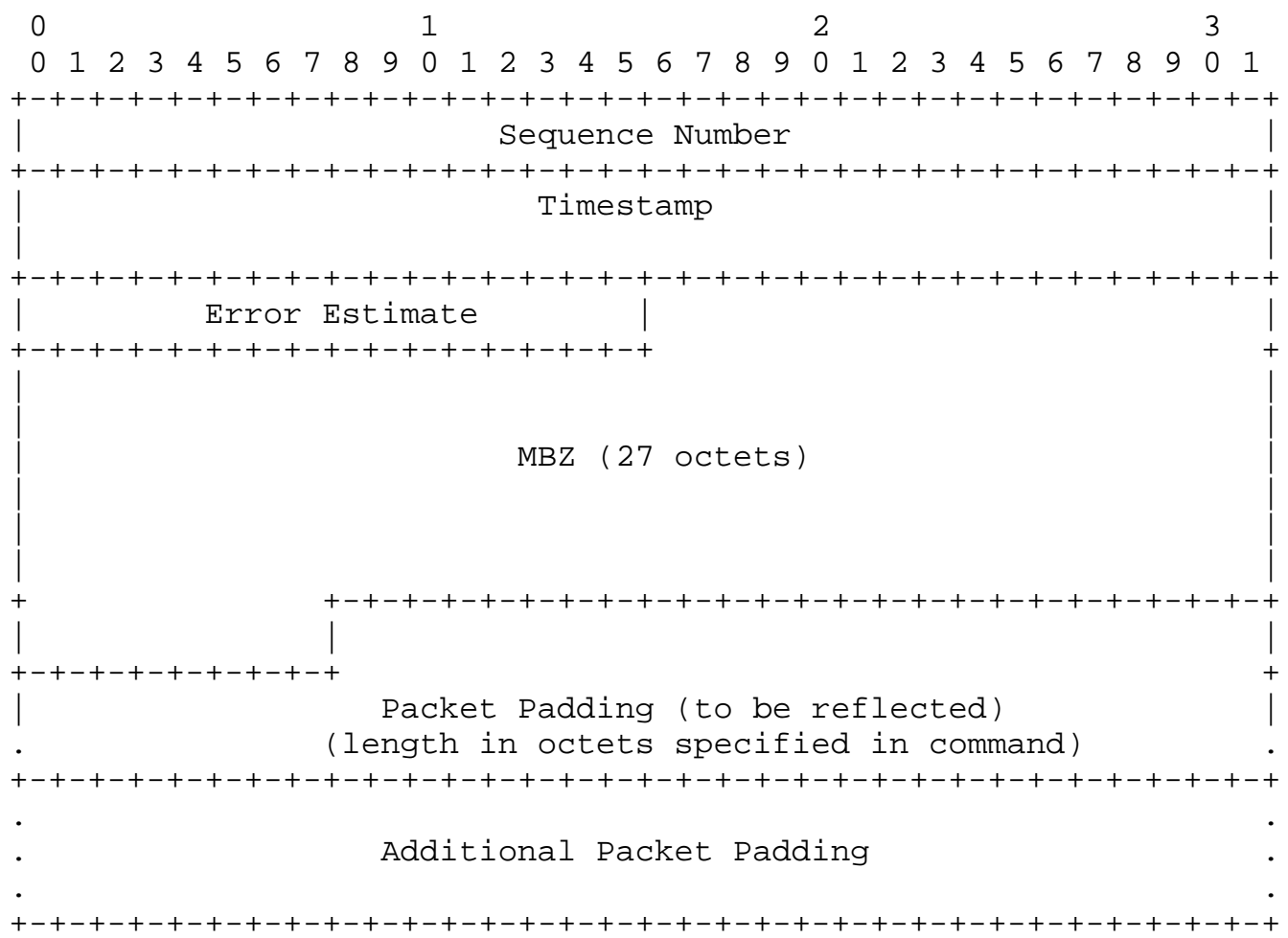

In this combined mode, the Packet Padding to be reflected follows the 27 MBZ octets. In Authenticated or Encrypted modes, the Packet Padding to be reflected follows the $56 \mathrm{MBZ}$ octets.

5.2. Reflector Behavior

The TWAMP Reflector follows the procedures and guidelines in Section 4.2 of [RFC5357], with the following additional functions:

- Reflect Octets mode: Designated octets in the Packet Padding (to be reflected) field of the Session-Sender's test packet MUST be included in the Session-Reflector's test packet. 
- Symmetrical Size mode: The Session-Reflector MUST operate using the Session_Reflector Packet Format defined in Section 5.1.4, where the Padding Octets are separated from the information fields.

\subsubsection{Reflect Octets: Session-Reflector Packet Format and Contents}

The Reflect Padding feature re-designates the Packet Padding field, as shown below. When the Reflect Octets mode is selected, the Session-Sender SHALL use the following TWAMP-Test Packet Format in Unauthenticated mode:

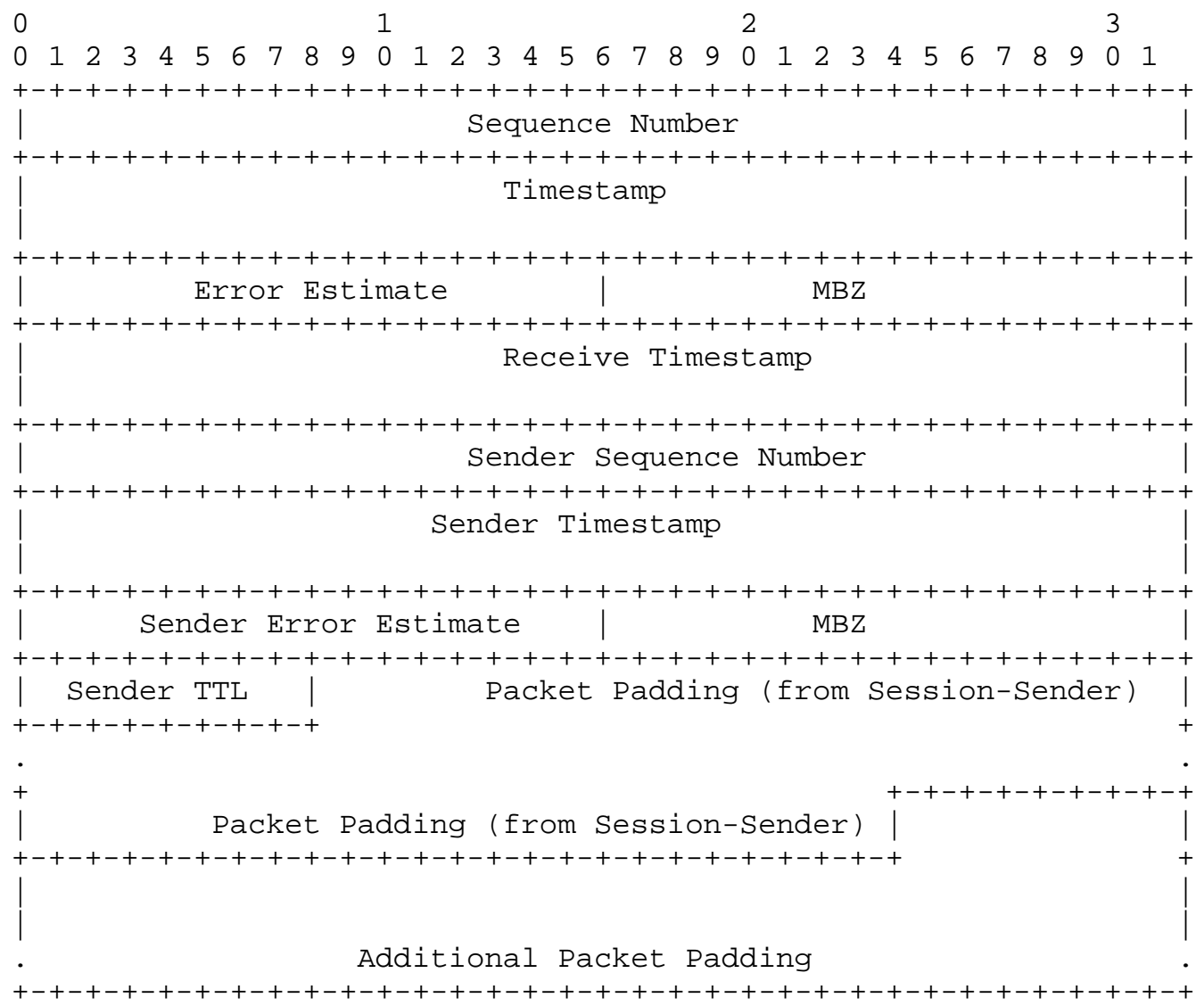

The Packet Padding (from Session-Sender) field MUST be the same octets as the Packet Padding (to be reflected) field in the SessionSender's test packet, and therefore MUST conform to the length specified in the Request-TW-Session message. 
When the Server has returned a non-zero value in the Server octets field of the Accept Session message (as described in Section 4.3), then the Session-Reflector SHALL reflect these octets the same as the rest of the Packet Padding (to be reflected) field.

Section 4.2.1 of [RFC5357] recommends a padding truncation process for use in TWAMP. When using that process in conjunction with the Reflect Octets mode, the Session-Reflector MUST reflect the designated octets from the Session-Sender's test packet in the Packet Padding (from Session-Sender) field, and MAY re-use additional Packet Padding from the Session-Sender. The Session-Reflector MUST truncate the padding such that the highest number octets are discarded, and the test packet length equals the Session-Sender's packet length. When using the recommended truncation process, the Session-Reflector MUST truncate exactly 27 octets of padding in Unauthenticated mode, and exactly 56 octets in Authenticated and Encrypted modes.

In any case, the Session-Reflector MAY re-use the Sender's Packet Padding (since the requirements for padding generation are the same for each).

5.2.2. Symmetrical Size: Session-Reflector Packet Format

When Symmetrical Size mode is selected, the Session-Reflector packet formats for Unauthenticated and Authenticated/Encrypted modes are identical to the core TWAMP specification, see Section 4.2.1 of [RFC5357]. Thus, the Session-Reflector test packet format is unchanged.

The Session-Reflector MUST construct its test packet using the information in the Session-Sender's test packet. The length of the Session-Reflector's test packet SHALL equal the length of the Session-Sender's test packet.

5.2.3. Symmetrical Size AND Reflect Octets: Session-Sender Packet Format

When both the Symmetrical Size mode and the Reflect Octets mode are selected, the Session-Reflector MUST operate using the Session-Sender Packet Format defined in Section 5.1.5, where the Padding Octets are separated from the information fields, and the Packet Padding (to be reflected) field precedes the Additional Padding.

The Session-Reflector SHALL use the same TWAMP-Test Packet Format as specified in Section 5.2.1, above. 
6. Security Considerations

These extended modes of operation do not appear to permit any new attacks on hosts communicating with core TWAMP [RFC5357].

The security considerations that apply to any active measurement of live networks are relevant here as well. See [RFC4656] and [RFC5357].

7. IANA Considerations

This memo adds two modes to the IANA registry for the TWAMP Modes field, and describes behavior when the new modes are used. This field is a recognized extension mechanism for TWAMP.

7.1. Registry Specification

IANA has created a TWAMP-Modes registry (as requested in [RFC5618]). TWAMP-Modes are specified in TWAMP Server Greeting messages and setup Response messages, as described in Section 3.1 of [RFC5357], consistent with section 3.1 of [RFC4656], and extended by this memo. Modes are indicated by setting bits in the 32-bit Modes field that correspond to values in the Modes registry. For the TWAMP-Modes registry, we expect that new features will be assigned increasing registry values that correspond to single bit positions, unless there is a good reason to do otherwise (more complex encoding than singlebit positions may be used in the future to access the $2^{\wedge} 32$ value space).

7.2. Registry Contents

The TWAMP-Modes registry has been augmented as follows:

$\begin{array}{lll}\text { Value } & \text { Description } & \text { Semantics Definition } \\ -32 & \text { Reflect Octets } & \text { this memo, Section } 3.1 \\ & \text { Capability } & \text { new bit position (5) } \\ 64 & \text { Symmetrical Size } & \text { this memo, Section } 3.1 \\ & \text { Sender Test Packet Format new bit position (6) }\end{array}$

8. Acknowledgements

The authors thank Steve Baillargeon, Walt Steverson, and Stina Ross for helpful review and comments. 
9. Normative References

[RFC2119] Bradner, S., "Key words for use in RFCs to Indicate Requirement Levels", BCP 14, RFC 2119, March 1997.

[RFC4656] Shalunov, S., Teitelbaum, B., Karp, A., Boote, J., and M. Zekauskas, "A One-way Active Measurement Protocol (OWAMP)", RFC 4656, September 2006.

[RFC5357] Hedayat, K., Krzanowski, R., Morton, A., Yum, K., and J. Babiarz, "A Two-Way Active Measurement Protocol (TWAMP)", RFC 5357, October 2008.

[RFC5618] Morton, A. and K. Hedayat, "Mixed Security Mode for the Two-Way Active Measurement Protocol (TWAMP)", RFC 5618, August 2009 .

Authors' Addresses

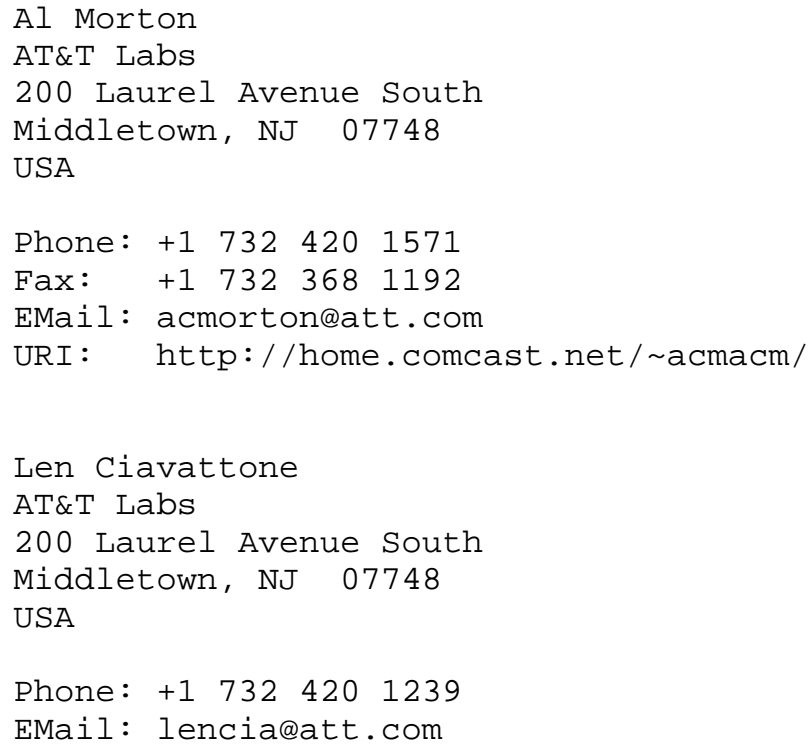


